\title{
Free Triiodothyronine Concentrations Are Inversely Associated with Microalbuminuria
}

\author{
Yulin Zhou, ${ }^{1}$ Lei Ye, ${ }^{1,2}$ Tiange Wang, ${ }^{1,2}$ Jie Hong, ${ }^{1}$ Yufang Bi, ${ }^{1,2}$ Jie Zhang, ${ }^{1,2}$ \\ Baihui Xu, ${ }^{1,2}$ Jichao Sun, ${ }^{1,2}$ Xiaolin Huang, ${ }^{1,2}$ and Min $\mathrm{Xu}^{1,2}$ \\ ${ }^{1}$ Shanghai Clinical Center for Endocrine and Metabolic Diseases, Shanghai Institute of Endocrine and Metabolic Diseases, \\ Department of Endocrinology and Metabolism, Rui-Jin Hospital, Shanghai Jiao-Tong University School of Medicine, \\ 197 Rui-Jin 2nd Road, Shanghai 200025, China \\ ${ }^{2}$ Key Laboratory for Endocrine and Metabolic Diseases of Ministry of Health, Rui-Jin Hospital, Shanghai Jiao-Tong \\ University School of Medicine, E-Institute of Shanghai Universities, Shanghai 200025, China
}

Correspondence should be addressed to Min Xu; della.xumin@163.com

Received 29 June 2014; Revised 27 October 2014; Accepted 28 October 2014; Published 16 November 2014

Academic Editor: Giorgio Iervasi

Copyright (c) 2014 Yulin Zhou et al. This is an open access article distributed under the Creative Commons Attribution License, which permits unrestricted use, distribution, and reproduction in any medium, provided the original work is properly cited.

Thyroid function and microalbuminuria are both associated with vascular disease and endothelial damage. However, whether thyroid function is associated with microalbuminuria is not well established. The objective was to explore the relationship between thyroid hormones and microalbuminuria in Chinese population. A community-based cross-sectional study was performed among 3,346 Chinese adults (aged $\geq 40$ years). Serum free triiodothyronine (FT3), free thyroxine (FT4), and TSH (thyroid stimulating hormone) were determined by chemiluminescent microparticle immunoassay. A single-void first morning urine sample was obtained for urinary albumin-creatinine ratio measurement. The prevalence of microalbuminuria decreased according to FT3 quartiles $(13.2,9.5,8.6$, and $8.2 \%, P$ for trend $=0.0005)$. A fully adjusted logistic regression analysis showed that high FT3 levels were associated with low prevalent microalbuminuria. The adjusted odds ratios for microalbuminuria were 0.61 (95\% CI, 0.43-0.87, $P=0.007)$ when comparing the highest with the lowest quartile of FT3. The exclusion of participants with abnormal FT3 did not appreciably change the results $(\mathrm{OR}=0.69,95 \% \mathrm{CI}, 0.49-0.98, P=0.02)$. We concluded that serum FT3 levels, even within the normal range, were inversely associated with microalbuminuria in middle-aged and elderly Chinese adults. FT3 concentrations might play a role in the pathogenesis of microalbuminuria.

\section{Introduction}

Microalbuminuria was first reported in diabetic patients by Viberti et al. in 1982 [1]. It was defined as the urine albumin-to-creatinine ratio (UACR) of 30 to $300 \mathrm{mg} / \mathrm{g}$ and originally used to predict chronic kidney disease and diabetic nephropathy [2]. Previous studies have confirmed that there is an increased risk of cardiovascular disease and death related to microalbuminuria [3]. The association between microalbuminuria, progressive atherosclerotic vascular disease, and renal damage has been well established in patients with systemic diseases [4]. Microalbuminuria is extensively believed to reflect a generalized impairment of the endothelium [5] which is an important determinant of vascular disease.
Thyroid hormones are also found to be associated with endothelial damage and cardiovascular disease [6-9]. Previous studies have shown that thyroid hormones, via dilation of blood vessel, production of vasodilator molecules, inhibition of angiotensin II receptor, and its signal transduction, regulate endothelial function and vascular homeostasis $[7,8]$. Moreover, in experimental models, triiodothyronine (T3) had important effects on the vascular system, such as inducing relaxation of vascular smooth muscle cells through a direct or indirect effect $[8,9]$. The epidemiology study of microalbuminuria also revealed a close association between systemic endothelial dysfunction and vascular disease [10]. However, evidence for the association between thyroid hormones and microalbuminuria in a community-based study, if any, is scarce. Just recently, Mervat and colleagues reported 
that subclinical hypothyroidism is independently associated with microalbuminuria in 147 prediabetic adults [11]. But the sample size was relatively small and it was not a communitybased study.

In this large community-based cross-sectional study, we aimed to investigate the relationship between thyroid hormones and microalbuminuria in middle-aged and elderly Chinese population.

\section{Subjects and Methods}

2.1. Study Population and Design. A cross-sectional survey was performed during 2008-2009 in a population from Songnan community located in Baoshan District, Shanghai. The selection criteria and design of the study have been described previously [12]. Briefly, in phase 1 (June and July 2008), we conducted the investigation on residents aged 40 years or older. A total of 10,185 participants were included in the screening examination. All participants received a fasting blood test. Based on the results of fasting glucose level and history of diabetes, participants were categorized into three groups: normal glucose regulation (NGR), defined as a fasting plasma glucose level less than $5.6 \mathrm{mmol} / \mathrm{L}(<101 \mathrm{mg} / \mathrm{dL})$ and no history of diabetes; impaired glucose regulation (IGR), defined as a fasting plasma glucose level of 5.6 to less than $7.0 \mathrm{mmol} / \mathrm{L}$ (101 to $<126 \mathrm{mg} / \mathrm{dL}$ ) and no history of diabetes; and diabetes, defined as a fasting plasma glucose level of $7.0 \mathrm{mmol} / \mathrm{L}$ or greater $(\geq 126 \mathrm{mg} / \mathrm{dL})$ or a history of diabetes. Because subjects with lower glucose levels were expected to have a lower participation rate than those with higher glucose levels, we randomly selected participants from these groups according to a ratio of 1.44 (NGR):1.2 (IGR):1 (DM) to receive a more comprehensive survey including thyroid function test, a standard 75-g OGTT, blood and urine collection, an anthropometric measurement, and a questionnaire survey in phase 2 (June through August 2009).

A total of 3455 persons with blood and urine samples were included in phase 2 survey. Exclusion criteria were (i) participants having a personal history of overt hyperthyroidism, hypothyroidism, or thyroiditis and having been taking thyroxine or antithyroid drugs $(n=12)$; (ii) taking medications affecting thyroid function such as amiodarone, lithium, antipsychotic drugs, and antiepileptic drugs $(n=$ $5)$; (iii) history of thyroidectomy $(n=2)$; (iv) participants with UACR $\geq 300 \mathrm{mg} / \mathrm{g}(n=34)$. Moreover, 56 participants without complete thyroid function or UACR data were also excluded from the study. Finally, a total of 3346 subjects (1336 men and 2010 women) remained in the present analysis. The participants (3346 subjects) and the nonparticipants (6839 subjects) were similar in characteristics, such as sex and age distribution.

This study was approved by the Institutional Review Board of the Rui-Jin Hospital, Shanghai Jiao Tong University School of Medicine, and was in accordance with the principle of the Helsinki Declaration II. The written informed consent was obtained from each participant.

2.2. Clinical and Biochemical Measurements. Sociodemographics, medical history, and lifestyles including smoking and drinking status were conducted by trained personnel. Current smoking was defined as "yes" if a subject smoked at least one cigarette per day or seven cigarettes per week in the past 6 months. Current alcohol drinking was defined as "yes" if a subject consumed alcohol at least once a week in the past 6 months. Weight, height, and blood pressure were measured by experienced nurses. Body mass index (BMI) was calculated using the formula of weight/height ${ }^{2}$ (kilograms per squared meters). Three sitting blood pressure measurements taken consecutively at $1 \mathrm{~min}$ interval after at least $5 \mathrm{~min}$ rest using an automated electronic device (OMRON model HEM-752 FUZZY; Omron Co., Dalian, China) were averaged for analysis. Hypertension was defined when systolic blood pressure (SBP) was above $140 \mathrm{mmHg}$, when diastolic blood pressure (DBP) was above $90 \mathrm{mmHg}$, or when taking medication for blood pressure control.

All participants were admitted after an overnight fast of $>10 \mathrm{~h}$ and two-point ( 0 and $2 \mathrm{~h}$ ) OGTT with a 75 g glucose load was performed. Plasma and sera were obtained. The fasting and postloading venous blood samples were collected into a routine tube, respectively, and were immediately centrifuged on site at $4^{\circ} \mathrm{C}$. Sera for the measurement of thyroid function were aliquot and kept frozen at $-80^{\circ} \mathrm{C}$ until use. Blood glucose, serum creatinine, lipid profile including total cholesterol (TC), triglycerides, high-density lipoprotein cholesterol (HDL-C), low-density lipoprotein cholesterol (LDL-C), and high-sensitivity C-reactive protein (hs-CRP) were measured within $1 \mathrm{~h}$ of collection with an automated biochemical instrument (Bayer Biochemical autoanalyzer ADVIA 1650, Bayer, Leverkusen, Germany). Hyperlipidemia was defined according to the National Cholesterol Education Program Adults Treatment Panel III (NCEP ATPIII) criteria [13]. The abbreviated Modification of Diet in Renal Disease (MDRD) Study formula as recalibrated for Chinese was used to calculate the estimated glomerular filtration rate (eGFR) expressed in $\mathrm{mL} / \mathrm{min} / 1.73 \mathrm{~m}^{2}: 186 \times($ serum creatinine value in $\mu \mathrm{mol} / \mathrm{L} \times 0.011)-1.154 \times($ age $)-0.203 \times(0.742$ if female $)$ $\times 1.233$ (the number 1.233 is the adjusting coefficient for Chinese) [14].

A first-voided, early-morning spot urine sample was collected for assessing the UACR within $1 \mathrm{~h}$ after sample collection. All participants were advised to refrain from vigorous exercise before providing the urine sample. Urinary albumin concentration was measured using immunoturbidimetry (Beijia Biochemistry, Shanghai, China), and urinary creatinine concentration was determined by a modified Jaffe method on an automatic analyzer (Beckman LX/20, Brea, CA). UACR was calculated as urinary albumin concentration divided by creatinine concentration and expressed in $\mathrm{mg} / \mathrm{g}$. Microalbuminuria was defined as the UACR ranges within $30-300 \mathrm{mg} / \mathrm{g}$ and macroalbuminuria as $\geq 300 \mathrm{mg} / \mathrm{g}[2,15]$.

2.3. Measurements of Thyroid Function. We measured thyroid stimulating hormone (TSH), free triiodothyronine (FT3), free thyroxine (FT4), thyroid peroxidase antibody (TPOAb), and thyroglobulin antibody (TgAb) levels. Thyroid function tests were analyzed in the Clinical Laboratory for Endocrinology, Shanghai Institution of Endocrine and 
Metabolic Diseases, which was certified by College of American Pathologists [16]. Serum FT3 (reference range: 2.62 to $6.49 \mathrm{pmol} / \mathrm{L}$ ), FT4 (reference range: 9.01 to $19.04 \mathrm{pmol} / \mathrm{L}$ ), and TSH (reference range: 0.35 to $4.94 \mu \mathrm{IU} / \mathrm{mL}$ ) were determined by chemiluminescent microparticle immunoassay method (Architect system; Abbott Laboratories, Abbott Park, IL). The coefficients of variations were $4.7-8.0 \%$ for FT3, 2.6-5.3\% for FT4, and 3.1-3.4\% for TSH. Serum anti-thyroid antibodies (TPOAb and TgAb) were measured by chemiluminescent microparticle immunoassay using Architect Anti-TPO and Anti-TG on the Architect i System (Abbott Laboratories). Reference ranges were TPOAb $<5.61 \mathrm{IU} / \mathrm{mL}$ (with an interassay $\mathrm{CV}$ of $4.3-6.8 \%$ ) and $\mathrm{TgAb}<4.11 \mathrm{IU} / \mathrm{mL}$ (with an interassay $\mathrm{CV}$ of $3.2-5.2 \%$ ).

2.4. Statistics Analysis. All of the statistical analyses were performed using SAS version 9.2 (SAS Institute, Cary, NC), and a $P$ value $<0.05$ (two-sided) indicated statistical significance. Continuous variables were summarized as means \pm $\mathrm{SD}$ or medians (interquartile ranges) for skewed variables. All categorical variables were presented as numbers and percentages. Triglycerides, fasting plasma glucose (FPG), OGTT 2-hour plasma glucose (OGTT-2 h PG), hs-CRP, FT3, FT4, TSH, TPOAb and TgAb, and UACR were normalized by logarithmically transformed before analyses because of nonnormal distribution.

The study participants were divided into four groups according to the quartiles of FT3. Demographic and metabolic features in each quartile were described and $P$ for trend is measured using linear regression analysis across FT3 quartiles.

To investigate the association of FT3 categories and microalbuminuria, univariate- and multivariate-adjusted logistic regression models were performed with microalbuminuria as the dependent variable and FT3 categories as the independent variable along with the potential confounding factors. In the multivariable logistic regression models, we adjusted for the potential confounding factors including age, sex, BMI, smoking and drinking status, SBP, and DBP, TC, triglycerides, FPG and OGTT-2 h PG, TPOAb and TgAb, hsCRP, use of angiotensin-converting enzyme inhibitor (ACEI) and/or angiotensin receptor blocker (ARB) drugs, and eGFR. Odds ratios (OR) with the lowest quartile were computed as the reference group.

To study if the association with FT3 and microalbuminuria was more prominent in some specific subgroups, we further performed stratified analyses on the associations between FT3 and the risk of microalbuminuria by the major risk factors including sex, age, BMI, hypertension, hyperlipidemia, diabetes according to OGTT diagnosis criteria, hsCRP, and eGFR. Odds ratios were calculated for an increase of each 1 s.d. in log FT3 concentration in subgroups of the strata variables. Interactions of each above strata variable with the association of serum $\log$ FT3 and microalbuminuria risks were tested by introducing the variable $\times \log$ FT3 into the univariate model.

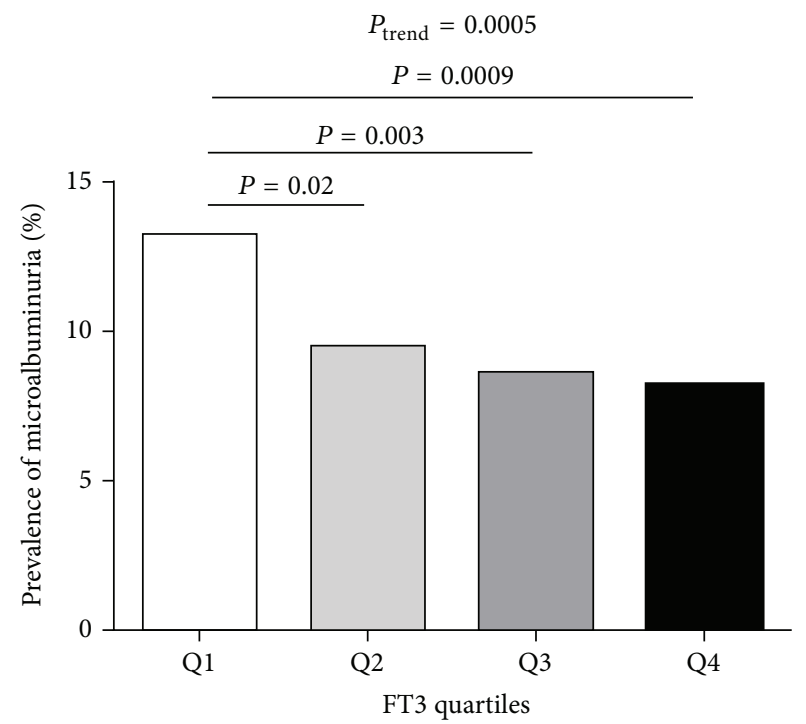

FIGURE 1: Prevalence of microalbuminuria in different FT3 quartiles: quartile $1(\mathrm{Q} 1, n=111), \leq 4.28 \mathrm{pmol} / \mathrm{L}$; quartile $2(\mathrm{Q} 2, n=80), 4.29$ $4.60 \mathrm{pmol} / \mathrm{L}$; quartile 3 (Q3, $n=73$ ), $4.61-4.93 \mathrm{pmol} / \mathrm{L}$; quartile 4 $(\mathrm{Q} 4, n=67),>4.94 \mathrm{pmol} / \mathrm{L}$.

\section{Results}

3.1. Characteristics of the Study Population. Among the 3,346 participants, the prevalence of microalbuminuria was $9.9 \%$ and no difference was found between males and females (9.2 versus $10.4 \%, P>0.05$ ). FT3 concentrations in male were significantly higher than that in female $(P<0.0001)$. Then participants were divided into four groups according to quartiles of FT3 with the first quartile representing the lowest one and the fourth quartile representing the highest one $(\leq 4.28,4.29-4.60,4.61-4.93$, and $>4.94 \mathrm{pmol} / \mathrm{L})$. The general characteristics of the four groups were presented in Table 1. Metabolic risk factors including sex distribution, current smoking or drinking status, BMI, DBP, triglycerides, TC, LDL-C, FT4, and eGFR increased significantly with the increment of FT3, whereas age, OGTT-2 h PG, HDL-C, TSH, and UACR decreased (all $P$ for trend $<0.05$ ). Compared with the participants in the lowest quartile of FT3, those in the second, the third, and the highest quartiles have significantly lower levels of UACR as follows: 6.08 (2.86$12.86)$ versus $5.93(2.70-12.39), 5.93(2.79-12.48)$, and 5.28 $(2.65-12.12) \mathrm{mg} / \mathrm{g}$ ( $P$ for trend $<0.05$, Table 1$)$. Nevertheless, no statistical difference was found for SBP, use of ACEI/ARB drugs, FPG, TgAb, TPOAb, and hs-CRP among the four groups.

3.2. Prevalence of Microalbuminuria in Different FT3 Levels. The prevalence of microalbuminuria differed in the four groups with different FT3 quartiles. From the lowest quartile across to the highest one of FT3 quartiles, the prevalence of microalbuminuria was $13.2,9.5,8.6$, and $8.2 \%$, respectively ( $P$ for trend $=0.0005$, Figure 1$)$. Compared to the lowest FT3 quartile, the second, the third, and the highest quartiles 
TABLE 1: General characteristics of the study population.

\begin{tabular}{|c|c|c|c|c|c|}
\hline & \multicolumn{5}{|c|}{ FT3 quartiles (pmol/L) } \\
\hline & $\begin{array}{l}\text { Quartile 1 } \\
(n=839)\end{array}$ & $\begin{array}{l}\text { Quartile } 2 \\
(n=843)\end{array}$ & $\begin{array}{l}\text { Quartile } 3 \\
(n=845)\end{array}$ & $\begin{array}{l}\text { Quartile } 4 \\
(n=819)\end{array}$ & $P$ for trend \\
\hline Male & $275(32.8)$ & $293(34.8)$ & $329(38.9)$ & $439(53.6)$ & $<0.0001$ \\
\hline Age (years) & $63.6 \pm 11.1$ & $61.0 \pm 10.1$ & $60.1 \pm 9.5$ & $58.4 \pm 8.3$ & $<0.0001$ \\
\hline BMI $\left(\mathrm{kg} / \mathrm{m}^{2}\right)$ & $24.9 \pm 3.7$ & $25.3 \pm 3.7$ & $25.3 \pm 3.8$ & $25.7 \pm 3.5$ & $<0.0001$ \\
\hline Current smokers & $120(14.3)$ & $154(18.3)$ & $163(19.3)$ & $251(30.7)$ & $<0.0001$ \\
\hline Current drinkers & $111(13.2)$ & $125(14.8)$ & $129(15.3)$ & $160(19.5)$ & 0.0006 \\
\hline Use of ACEI/ARB drugs & $33(3.93)$ & $32(3.80)$ & $31(3.67)$ & $33(4.03)$ & 0.98 \\
\hline $\mathrm{SBP}(\mathrm{mmHg})$ & $139.5 \pm 23.5$ & $137.6 \pm 22.2$ & $138.6 \pm 21.2$ & $138.9 \pm 20.4$ & 0.77 \\
\hline DBP (mmHg) & $76.3 \pm 10.1$ & $77.8 \pm 10.2$ & $78.6 \pm 9.6$ & $81.0 \pm 10.5$ & $<0.0001$ \\
\hline FPG (mmol/L) & $5.2(4.7-6.1)$ & $5.2(4.8-6.1)$ & $5.2(4.8-5.8)$ & $5.3(4.8-6.0)$ & 0.08 \\
\hline OGTT-2 h PG (mmol/L) & $8.5(6.3-14.4)$ & $7.8(6.1-12.5)$ & $7.6(6.1-11.4)$ & $7.7(6.3-11.4)$ & $<0.0001$ \\
\hline Triglycerides (mmol/L) & $1.28(0.89-1.89)$ & $1.40(1.00-2.02)$ & $1.46(1.01-2.14)$ & $1.61(1.10-2.34)$ & $<0.0001$ \\
\hline TC (mmol/L) & $5.07 \pm 1.04$ & $5.15 \pm 0.98$ & $5.21 \pm 0.93$ & $5.17 \pm 0.99$ & 0.02 \\
\hline $\mathrm{HDL}-\mathrm{C}(\mathrm{mmol} / \mathrm{L})$ & $1.37 \pm 0.33$ & $1.36 \pm 0.29$ & $1.37 \pm 0.32$ & $1.32 \pm 0.29$ & 0.01 \\
\hline LDL-C (mmol/L) & $2.34 \pm 0.71$ & $2.41 \pm 0.70$ & $2.39 \pm 0.66$ & $2.42 \pm 0.68$ & 0.03 \\
\hline FT3 (pmol/L) & $4.05(3.84-4.18)$ & $4.45(4.37-4.53)$ & $4.76(4.68-4.85)$ & $5.18(5.05-5.38)$ & - \\
\hline FT4 (pmol/L) & $13.7(12.7-14.9)$ & $14.1(13.2-15.1)$ & $14.2(13.3-15.3)$ & $14.8(13.8-15.8)$ & $<0.0001$ \\
\hline TSH $(\mu \mathrm{IU} / \mathrm{mL})$ & $1.59(1.07-2.33)$ & $1.50(1.02-2.18)$ & $1.44(1.01-2.160$ & $1.35(0.92-1.99)$ & $<0.0001$ \\
\hline TPOAb (IU/mL) & $0.30(0.17-0.74)$ & $0.31(0.17-0.77)$ & $0.30(0.16-0.72)$ & $0.34(0.18-0.81)$ & 0.93 \\
\hline $\operatorname{TgAb}(\mathrm{IU} / \mathrm{mL})$ & $1.09(0.70-2.73)$ & $1.08(0.70-2.95)$ & $1.02(0.71-2.27)$ & $1.05(0.72-2.25)$ & 0.08 \\
\hline eGFR $\left(\mathrm{mL} / \mathrm{min} / 1.73 \mathrm{~m}^{2}\right)$ & $107.5 \pm 28.6$ & $112.6 \pm 24.4$ & $112.8 \pm 24.9$ & $115.8 \pm 22.9$ & $<0.0001$ \\
\hline UACR (mg/g) & $6.08(2.86-12.86)$ & $5.93(2.70-12.39)$ & $5.93(2.79-12.48)$ & $5.28(2.65-12.12)$ & 0.03 \\
\hline Hs-CRP (mg/g) & $0.21(0.05-1.13)$ & $0.18(0.06-0.85)$ & $0.18(0.07-0.61)$ & $0.24(0.08-0.82)$ & 0.58 \\
\hline
\end{tabular}

Data were means \pm SD or medians (interquartile range) or numbers (proportions). BMI, body mass index; ACEI, angiotensin-converting enzyme inhibitor; ARB, angiotensin receptor blocker; SBP, systolic blood pressure; DBP, diastolic blood pressure; TC, total cholesterol; HDL-C, high-density lipoprotein cholesterol; LDL-C, low-density lipoprotein; FPG, fasting plasma glucose; OGTT-2 h PG, OGTT 2-hour plasma glucose; FT3, free triiodothyronine; FT4, free thyroxine; TSH, thyroid stimulating hormone; TPOAb, thyroid peroxidase antibody; TgAb, thyroglobulin antibody; eGFR, estimate glomerular filtration rate; UACR, urinary albumin-to-creatinine ratio.

FT3 quartiles were as follows: quartile 1, $\leq 4.28 \mathrm{pmol} / \mathrm{L}$; quartile 2, 4.29-4.60 pmol/L; quartile 3, 4.61-4.93 pmol/L; quartile 4, $>4.94 \mathrm{pmol} / \mathrm{L}$.

showed a significant decrease in the prevalence of microalbuminuria $(P=0.02,0.003$, and 0.0009 , resp.).

3.3. Association between FT3 and Microalbuminuria. In the univariable logistic regression model, the risk for microalbuminuria decreased across FT3 quartiles. The odds ratio (OR) for the highest FT3 quartile compared with the lowest quartile was $0.58,95 \%$ confidence intervals (95\% CI) $0.42-0.81, P=$ 0.001 , and those in the second and third quartile were also less likely to have microalbuminuria (OR: 0.69, 95\% CI, 0.51-0.93; $P=0.02$ and OR: $0.62,95 \% \mathrm{CI}, 0.45-0.85 ; P=0.003$ resp.). The test for the trend was significant $(P$ for trend $=0.0006)$. After adjusting for age, sex, BMI, and smoking and drinking status, participants in the second, third, and highest quartiles were still less likely to have microalbuminuria compared with those in the lowest quartile (adjusted OR: 0.72, 95\% CI, 0.52-0.99, $P=0.04 ; 0.66,95 \% \mathrm{CI}, 0.48-0.91, P=$ 0.01 ; and $0.58,95 \% \mathrm{CI}, 0.41-0.82, P=0.002$, resp.). After further adjustments for other potential confounding factors including SBP, DBP, TC, triglycerides, FPG and OGTT-2 h PG, TPOAb and TgAb, hs-CRP, use of ACEI/ARB drugs, and eGFR, a fully adjusted logistic regression (Table 2) showed similar associations for the third and highest quartile but not for the second quartile (adjusted ORs for the highest versus the lowest quartile: $0.61,95 \% \mathrm{CI}, 0.43-0.87, P=$ 0.007 , and for the third versus the lowest quartile: $0.70,95 \%$ CI, 0.50-0.98, $P=0.04)$. The tests for the trend in the multivariable analyses were all significant ( $P$ for trend 0.01 ) (Table 2). We also conducted linear regression analyses for FT3 and UACR. In both simple and multivariate-adjusted linear regression analyses, FT3 levels were negatively and significantly associated with UACR $(P$ values $<0.05)$.

Then we excluded the participants with abnormal FT3 levels (FT3 $<2.62$ or $\geq 6.49 \mathrm{pmol} / \mathrm{L}, n=41$ ). The same analysis was performed for associations between FT3 and microalbuminuria. We found a similar inverse association between serum FT3 and microalbuminuria in unadjusted, adjusted, and fully adjusted model (Table 2). Compared with those in the lowest quartile, highest quartiles were still less likely to have microalbuminuria (OR 0.69; 95\% CI, 0.49-0.98, $P=0.04$ ) (Table 2). FT4 and TSH were not significantly associated with microalbuminuria in all the models generated (data not shown).

Furthermore, stratified analyses for multivariate-adjusted OR of microalbuminuria with each 1 s.d. increment in log 
TABLE 2: The risk of microalbuminuria according to quartiles of FT3.

\begin{tabular}{|c|c|c|c|c|c|}
\hline & \multicolumn{5}{|c|}{ FT3 quartiles (pmol/L) } \\
\hline & Quartile 1 & Quartile 2 & Quartile 3 & Quartile 4 & $P$ for trend \\
\hline \multicolumn{6}{|c|}{ In total participants $(n=3346)$} \\
\hline Cases/participants & $111 / 839$ & $80 / 843$ & $73 / 845$ & $67 / 819$ & \\
\hline Model 1 & 1.00 & $0.69(0.51-0.93)$ & $0.62(0.45-0.85)$ & $0.58(0.42-0.81)$ & 0.0006 \\
\hline Model 2 & 1.00 & $0.72(0.52-0.99)$ & $0.66(0.48-0.91)$ & $0.58(0.41-0.82)$ & 0.005 \\
\hline Model 3 & 1.00 & $0.74(0.53-1.02)$ & $0.70(0.50-0.98)$ & $0.61(0.43-0.87)$ & 0.007 \\
\hline \multicolumn{6}{|c|}{ In participants with normal FT3 levels $(n=3305)$} \\
\hline Cases/participants & $111 / 831$ & $79 / 841$ & $65 / 822$ & $73 / 811$ & \\
\hline Model 1 & 1.00 & $0.67(0.50-0.91)$ & $0.56(0.40-0.77)$ & $0.64(0.47-0.88)$ & 0.002 \\
\hline Model 2 & 1.00 & $0.71(0.51-0.97)$ & $0.60(0.43-0.84)$ & $0.65(0.46-0.91)$ & 0.02 \\
\hline Model 3 & 1.00 & $0.73(0.52-1.01)$ & $0.64(0.45-0.91)$ & $0.69(0.49-0.98)$ & 0.02 \\
\hline
\end{tabular}

See Table 1 for the FT3 quartiles definition. Data are odds ratios (ORs, 95\% confidential interval).

Model 1 is unadjusted.

Model 2 is adjusted for age, sex, BMI, and smoking and drinking status.

Model 3 is adjusted for age, sex, BMI, smoking and drinking status, SBP and DBP, TC, triglycerides, FPG and OGTT-2 h PG, TPOAb and TgAb, hs-CRP, use of ACEI/ARB drugs, and eGFR.

FT3 concentration in different subgroups were conducted. The associations between microalbuminuria and FT3 were not consistently the same in subgroups. Inverse associations between microalbuminuria and FT3 were significant in both age strata ( $<60$ and $\geq 60$ years), both BMI strata ( $<24$ and $\geq 24 \mathrm{~kg} / \mathrm{m}^{2}$ ), both hypertension strata (yes and no), both diabetes strata according to the OGTT diagnosis criteria (yes and no), and both hs-CRP strata ( $<3$ and $\geq 3 \mathrm{mg} / \mathrm{L}$ ). Inverse associations between microalbuminuria and FT3 were also persisted in male, those without hyperlipidemia and eGFR $\geq 90 \mathrm{~mL} / \mathrm{min}$ per $1.73 \mathrm{~m}^{2}$. No significant associations were detected in female, those with hyperlipidemia and eGFR < $90 \mathrm{~mL} / \mathrm{min}$ per $1.73 \mathrm{~m}^{2}$ (Figure 2). Nevertheless, interactions between FT3 and these risk factors were not significant $(P>$ 0.05) (Figure 2).

\section{Discussion}

In this community-based cross-sectional study, we found serum FT3 levels were inversely associated with microalbuminuria which is a powerful predictor of generalized vascular endothelial impairment. Moreover, the association was independent of other traditional vascular risk factors.

Microalbuminuria is a marker of increased risk of cardiovascular $(\mathrm{CV})$ and renal morbidity and mortality in diabetic subjects. Meanwhile, microalbuminuria is a marker of increased risk for diabetes mellitus, hypertension, deterioration of renal function, and CV morbidity and all-cause mortality in nondiabetic subjects [5]. The prevalence of microalbuminuria is relatively high in previous studies. Jones et al. [17] reported the prevalence of microalbuminuria in 22,244 subjects aged from 6 to $>80$ years to be $7.8 \%(6.1 \%$ in males and $9.7 \%$ in females) with progressively increasing prevalence in adults $>40$ years of age from the Third National Health and Nutritional Examination Survey (NHANES). Our data showed the prevalence of microalbuminuria was $9.9 \%$ (9.2\% in males versus $10.4 \%$ in females) in participants aged
40 or above. Microalbuminuria is regarded as an integrated marker of systemic endothelial dysfunction and vascular disease via strong associations with blood pressure levels, metabolic status, lipids, and smoking habits [18]. In our study, we confirmed participants with microalbuminuria were more likely to have higher levels of BMI, SBP and DBP, FPG, OGTT-2 h PG, TC, triglycerides and hs-CRP (all $P<0.05$ ), and lower levels of HDL-C (all $P<0.01)$ compared to those normoalbuminuria.

Thyroid hormone has crucial effects on the vascular system [19] and is also found to be associated with endothelial damage [20, 21]. Endothelial impairment has well-known effects on the development of hypertension, coronary artery disease, chronic heart failure, peripheral artery disease, diabetes, and chronic renal failure [22]. Serum FT3 level has been shown to be associated with impaired endothelial function as assessed by flow-mediated dilation in patients with chronic kidney disease [23]. By now no large-scale population-based studies have been published to focus on the association with thyroid function and microalbuminuria. Only one study [11] found an association between subclinical hypothyroidism and microalbuminuria in 147 prediabetic subjects. However, their sample size was small and the study was not population-based. In our study, after adjustment for a wide spectrum of lifestyle and biochemical risk factors we found FT3 was inversely associated with microalbuminuria in a large population. In addition, the association remained even in participants within normal FT3 level. These findings raised the possibility that normal thyroid function within the higher range was related to lower prevalence of microalbuminuria.

However, the exact mechanisms for inverse associations between FT3 and microalbuminuria are not well defined. Thyroid hormones have profound effects on vascular and endothelial functions [19-21]. Patients with subclinical hypothyroidism have endothelial dysfunction characterized by reduced endothelium-dependent vasodilatation and impaired nitric oxide availability. Moreover, this abnormal vascular profile is partially independent of dyslipidemia 


\begin{tabular}{|c|c|c|c|c|}
\hline & & OR (95\% CI) & $P$ value & $\begin{array}{c}P \text { for } \\
\text { interaction }\end{array}$ \\
\hline Total & $\longrightarrow$ & $0.85(0.76-0.96)$ & 0.007 & \\
\hline \multicolumn{5}{|l|}{ Sex } \\
\hline Male & 1 & $0.70(0.57-0.85)$ & 0.0003 & 0.8 \\
\hline Female & $\begin{array}{l}0 \\
\end{array}$ & $0.89(0.76-1.05)$ & 0.16 & \\
\hline \multicolumn{5}{|l|}{ Age (years) } \\
\hline$<60$ & $\longrightarrow$ & $0.82(0.68-1.00)$ & 0.04 & 0.15 \\
\hline$\geq 60$ & $\longrightarrow$ & $0.82(0.69-0.97)$ & 0.02 & \\
\hline \multicolumn{5}{|l|}{ BMI $\left(\mathrm{kg} / \mathrm{m}^{2}\right)$} \\
\hline$<24$ & - & $0.74(0.60-0.94)$ & 0.008 & 0.95 \\
\hline$\geq 24$ & - & $0.84(0.73-0.98)$ & 0.03 & \\
\hline \multicolumn{5}{|l|}{ Hypertension } \\
\hline No & - & $0.78(0.62-0.99)$ & 0.03 & 0.47 \\
\hline Yes & - & $0.84(0.72-0.96)$ & 0.01 & \\
\hline \multicolumn{5}{|l|}{ Diabetes } \\
\hline No & - & $0.83(0.70-0.99)$ & 0.03 & 0.26 \\
\hline Yes & - & $0.75(0.62-0.91)$ & 0.003 & \\
\hline \multicolumn{5}{|l|}{ Hyperlipidemia } \\
\hline No & - & $0.76(0.63-0.92)$ & 0.003 & 0.78 \\
\hline Yes & $\begin{array}{l}\vdots \\
\vdots\end{array}$ & $0.89(0.75-1.04)$ & 0.15 & \\
\hline \multicolumn{5}{|l|}{ Hs-CRP (mg/L) } \\
\hline$<3.0$ &  & $0.83(0.73-0.96)$ & 0.01 & 0.2 \\
\hline$\geq 3.0$ & $\bullet$ & $0.69(0.49-0.96)$ & 0.03 & \\
\hline \multicolumn{5}{|l|}{$\mathrm{eGFR}\left(\mathrm{mL} / \mathrm{min} / 1.73 \mathrm{~m}^{2}\right)$} \\
\hline$<90$ & $\vdots$ & $0.78(0.60-1.01)$ & 0.06 & 0.09 \\
\hline \multirow[t]{3}{*}{$\geq 90$} & 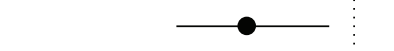 & $0.83(0.72-0.96)$ & 0.01 & \\
\hline & 0.5 & $\overrightarrow{1.5}$ & & \\
\hline & Odds ratios for microalbuminuria & & & \\
\hline
\end{tabular}

FIGURE 2: Adjusted odds ratios (OR) for each 1 s.d. increase in log FT3 concentration associated with the risk of microalbuminuria. Logistic regression model was adjusted for potential confounding factors including age, sex, BMI, smoking and drinking status, SBP and DBP, TC, triglycerides, FPG and OGTT-2 h PG, TPOAb and TgAb, hs-CRP, use of ACEI/ARB drugs, and eGFR (except for the strata variables). CI, confidence interval.

and restored by levothyroxine treatment [7, 24]. Vascular function is impaired in patients with mild and subclinical hypothyroidism, as documented by the increase in systemic vascular resistance and arterial stiffness and by the impaired endothelial function. The previous study showed the T3induced decrease in systemic vascular resistance may be explained by direct modulation of endothelium-dependent and -independent vasoregulation [9]. In experimental models, it was also shown that FT3 had antiatherosclerotic effects on the vascular bed via its effect on mitochondrial oxidation systems, induction of vasodilatatory molecules, and inhibition of angiotensin II receptor expression and downstream signal transduction $[8,25]$. Thus, endothelial damage/dysfunction, closely associated with the pathogenesis of atherosclerosis [26], impairs endothelium-dependent vasodilatation [27] which may mediate with microalbuminuria. It is highly possible that low FT3 could be a marker of endothelial dysfunction similar to presence of microalbuminuria and both might be mediated by unmeasured or unknown factor.

In addition, thyroid hormone is closely associated with the metabolic syndrome (Mets), a syndrome of insulin resistance, obesity, hypertension, and dislipidemia. The data from NHANES III showed strong positive associations between microalbuminuria and Mets [28]. Meanwhile, insulin resistance is generally hypothesized to be the major underlying pathophysiological process of Mets [29]. Low thyroid function (even in the euthyroid state) was reported to be associated with increased insulin resistance [30], which might mediate the association with microalbuminuria [31].

Microalbuminuria has been generally regarded as a marker of generalized vascular endothelial impairment [32]. Xiang et al. [33] reported endothelial dysfunction exists in euthyroidism patients with Hashimoto's disease and 
autoimmune reactivity, indicating TPOAb might be responsible for the endothelial dysfunction and the subsequent microalbuminuria. However, our data showed the link with microalbuminuria was roughly similar for FT3 with and without TPOAb. This demonstrates that the effect of FT3 on microalbuminuria might be independent of thyroid autoimmunity. Additionally, increasing evidence shows that systemic and vascular low-grade inflammation, as reflected by increased serum concentration of hs-CRP, may provide a mechanism for the pathogenesis of microalbuminuria [34]. However, we found FT3 inversely associated with microalbuminuria either in subjects with hs-CRP $\geq 3.0 \mathrm{mg} / \mathrm{L}$ or $<3.0 \mathrm{mg} / \mathrm{L}$, indicating that the association between FT3 and microalbuminuria cannot be explained by systemic inflammation.

Our study was the first to report an independent association between FT3 concentration and microalbuminuria in a large population. Our study provided confirmatory evidence for the association between FT3 and microalbuminuria. However, limitations are unavoidable. Firstly, our study was cross-sectional designed, and no causal relationships can be established. Further longitudinal research is required to clarify the cause and effect relationships. Secondly, the urinary albumin excretion was measured on a morning spoturine sample. We acknowledge that a $24 \mathrm{~h}$ urine sample would provide more stable measures for urinary albumin excretion. However, it was reported that the use of spot specimens for urinary albumin-to-creatinine ratio was more convenient for a large cohort survey and the results correlated well with $24 \mathrm{~h}$ urinary albumin excretion and could be used as a reliable alternative to $24 \mathrm{~h}$ urinary albumin excretion in large epidemiological research [35]. Thirdly, our study included only middle-aged and elderly subjects, and the results may not be applied to a general population with different age composition.

\section{Conclusions}

In summary, the present study shows inverse correlation between microalbuminuria, an established marker of generalized vascular endothelial impairment, and serum FT3. FT3 concentrations may play a role in the pathogenesis of microalbuminuria. Moreover, this inverse association also exists in subjects classified as being euthyroid, which implicates low normal thyroid function could adversely affect microalbuminuria even in euthyroidism. We therefore propose to consider FT3 levels in addition to Mets and other risk factors that are traditionally associated with microalbuminuria [5]. Further studies are needed to explore the mechanisms responsible for the correlation between FT3 and microalbuminuria.

\section{Conflict of Interests}

The authors declare that there is no conflict of interests regarding the publication of this paper.

\section{Acknowledgments}

The present study would not have been possible without the participation of the subjects. This study was supported by the grants from the Key Laboratory for Endocrine and Metabolic Diseases of Ministry of Health (1994DP131044), the National Clinical Research Center for Metabolic Disease of Ministry of Health (2013BAI09B13), the National Key New Drug Creation and Manufacturing Program of Ministry of Science and Technology (2012ZX09303006-001), and the Nature Science Foundation of China (no. 81270877).

\section{References}

[1] G. C. Viberti, R. J. Jarrett, R. D. Hill, A. Argyropoulos, U. Mahmud, and H. Keen, "Microalbuminuria as a predictor of clinical nephropathy in insulin-dependent diabetes mellitus," The Lancet, vol. 1, no. 8287, pp. 1430-1432, 1982.

[2] H. C. Gerstein, J. F. E. Mann, J. Pogue et al., "Prevalence and determinants of microalbuminuria in high-risk diabetic and nondiabetic patients in the heart outcomes prevention evaluation study," Diabetes Care, vol. 23, no. 2, pp. B35-B39, 2000.

[3] S. Romundstad, J. Holmen, H. Hallan, K. Kvenild, and H. Ellekjær, "Microalbuminuria and all-cause mortality in treated hypertensive individuals: does sex matter? The Nord-Trøndelag Health Study (HUNT), Norway," Circulation, vol. 108, no. 22, pp. 2783-2789, 2003.

[4] G. F. M. Strippoli, M. Craig, J. J. Deeks, F. P. Schena, and J. C. Craig, "Effects of angiotensin converting enzyme inhibitors and angiotensin II receptor antagonists on mortality and renal outcomes in diabetic nephropathy: systematic review," British Medical Journal, vol. 329, no. 7470, pp. 828-831, 2004.

[5] J.-M. Halimi, S. Hadjadj, V. Aboyans et al., "Microalbuminuria and urinary albumin excretion: French clinical practice guidelines," Diabetes \& Metabolism, vol. 33, no. 4, pp. 303-309, 2007.

[6] G. Pasqualetti, S. Tognini, A. Polini, N. Caraccio, and F. Monzani, "Is subclinical hypothyroidism a cardiovascular risk factor in the elderly?" Journal of Clinical Endocrinology and Metabolism, vol. 98, no. 6, pp. 2256-2266, 2013.

[7] S. Taddei, N. Caraccio, A. Virdis et al., "Impaired endotheliumdependent vasodilatation in subclinical hypothyroidism: beneficial effect of levothyroxine therapy," Journal of Clinical Endocrinology and Metabolism, vol. 88, no. 8, pp. 3731-3737, 2003.

[8] K. Fukuyama, T. Ichiki, K. Takeda et al., "Downregulation of vascular angiotensin II type 1 receptor by thyroid hormone," Hypertension, vol. 41, no. 3, pp. 598-603, 2003.

[9] B. Biondi, "Cardiovascular effects of mild hypothyroidism," Thyroid, vol. 17, no. 7, pp. 625-630, 2007.

[10] A. Singh and S. C. Satchell, "Microalbuminuria: causes and implications," Pediatric Nephrology, vol. 26, no. 11, pp. 1957-1965, 2011.

[11] M. M. El-Eshmawy, H. A. Abd El-Hafez, W. O. El Shabrawy, and I. A. Abdel Aal, "Subclinical hypothyroidism is independently associated with microalbuminuria in a cohort of prediabetic Egyptian adults," Diabetes and Metabolism Journal, vol. 37, no. 6, pp. 450-457, 2013.

[12] T. Wang, M. Li, B. Chen et al., "Urinary bisphenol A (BPA) concentration associates with obesity and insulin resistance," Journal of Clinical Endocrinology and Metabolism, vol. 97, no. 2, pp. e223-e227, 2012. 
[13] Expert Panel on Detection, Evaluation, and Treatment of High Blood Cholesterol in Adults, "Executive summary of the third report of the National Cholesterol Education Program (NCEP) expert panel on detection, evaluation, and treatment of high blood cholesterol in adults (adult treatment panel III)," The Journal of the American Medical Association, vol. 285, no. 19, pp. 2486-2497, 2001.

[14] Y.-C. Ma, L. Zuo, J.-H. Chen et al., "Modified glomerular filtration rate estimating equation for Chinese patients with chronic kidney disease," Journal of the American Society of Nephrology, vol. 17, no. 10, pp. 2937-2944, 2006.

[15] M. Li, M. Xu, Y. Bi et al., "Association between higher serum fetuin - a concentrations and abnormal albuminuria in middleaged and elderly Chinese with normal glucose tolerance," Diabetes Care, vol. 33, no. 11, pp. 2462-2464, 2010.

[16] T. Wang, J. Lu, M. Xu et al., "Urinary bisphenol a concentration and thyroid function in Chinese adults," Epidemiology, vol. 24, no. 2, pp. 295-302, 2013.

[17] C. A. Jones, M. E. Francis, M. S. Eberhardt et al., "Microalbuminuria in the US population: third National Health and Nutrition Examination Survey," American Journal of Kidney Diseases, vol. 39, no. 3, pp. 445-459, 2002.

[18] R. Pedrinelli, G. Dell'Omo, G. Penno, and M. Mariani, "Nondiabetic microalbuminuria, endothelial dysfunction and cardiovascular disease," Vascular Medicine, vol. 6, no. 4, pp. 257264, 2001.

[19] N. Rodondi, W. P. J. den Elzen, D. C. Bauer et al., "Subclinical hypothyroidism and the risk of coronary heart disease and mortality," The Journal of the American Medical Association, vol. 304, no. 12, pp. 1365-1374, 2010.

[20] B. Biondi and D. S. Cooper, "The clinical significance of subclinical thyroid dysfunction," Endocrine Reviews, vol. 29, no. 1, pp. 76-131, 2008.

[21] E. Tatar, F. Kircelli, G. Asci et al., "Associations of triiodothyronine levels with carotid atherosclerosis and arterial stiffness in hemodialysis patients," Clinical Journal of the American Society of Nephrology, vol. 6, no. 9, pp. 2240-2246, 2011.

[22] D. H. Endemann and E. L. Schiffrin, "Endothelial dysfunction," Journal of the American Society of Nephrology, vol. 15, no. 8, pp. 1983-1992, 2004.

[23] M. I. Yilmaz, A. Sonmez, M. Karaman et al., "Low triiodothyronine alters flow-mediated vasodilatation in advanced nondiabetic kidney disease," American Journal of Nephrology, vol. 33, no. 1, pp. 25-32, 2011.

[24] B. Biondi, M. Galderisi, L. Pagano et al., "Endothelial-mediated coronary flow reserve in patients with mild thyroid hormone deficiency," European Journal of Endocrinology, vol. 161, no. 2, pp. 323-329, 2009.

[25] K. Fukuyama, T. Ichiki, I. Imayama et al., "Thyroid hormone inhibits vascular remodeling through suppression of cAMP response element binding protein activity," Arteriosclerosis, Thrombosis, and Vascular Biology, vol. 26, no. 9, pp. 2049-2055, 2006.

[26] S. Taddei and A. Salvetti, "Endothelial dysfunction in essential hypertension: clinical implications," Journal of Hypertension, vol. 20, no. 9, pp. 1671-1674, 2002.

[27] P. Ruggenenti and G. Remuzzi, “Time to abandon microalbuminuria?” Kidney International, vol. 70, no. 7, pp. 1214-1222, 2006.

[28] L. Palaniappan, M. Carnethon, and S. P. Fortmann, "Association between microalbuminuria and the metabolic syndrome:
NHANES III," American Journal of Hypertension, vol. 16, no. 11, pp. 952-958, 2003.

[29] R. H. Eckel, S. M. Grundy, and P. Z. Zimmet, "The metabolic syndrome," The Lancet, vol. 365, no. 9468, pp. 1415-1428, 2005.

[30] J. D. J. Garduño-Garcia, U. Alvirde-Garcia, G. López-Carrasco et al., "TSH and free thyroxine concentrations are associated with differing metabolic markers in euthyroid subjects," European Journal of Endocrinology, vol. 163, no. 2, pp. 273-278, 2010.

[31] S. de Cosmo, A. Minenna, O. Ludovico et al., "Increased urinary albumin excretion, insulin resistance, and related cardiovascular risk factors in patients with type 2 diabetes: evidence of a sex-specific association," Diabetes Care, vol. 28, no. 4, pp. 910915, 2005.

[32] R. Pedrinelli, O. Giampietro, F. Carmassi et al., "Microalbuminuria and endothelial dysfunction in essential hypertension," The Lancet, vol. 344, no. 8914, pp. 14-18, 1994.

[33] G.-D. Xiang, Y.-S. He, L.-S. Zhao, J. Hou, L. Yue, and H.-J. Xiang, "Impairment of endothelium-dependent arterial dilation in Hashimoto's thyroiditis patients with euthyroidism," Clinical Endocrinology, vol. 64, no. 6, pp. 698-702, 2006.

[34] L. Jiang, W. Huang, Y. Liang et al., "Metabolic syndrome, Creactive protein and microalbuminuria in a rural Chinese population: a cross-sectional study," BMC Nephrology, vol. 14, no. 1, article 118, 2013.

[35] G. Eknoyan, T. Hostetter, G. L. Bakris et al., "Proteinuria and other markers of chronic kidney disease: a position statement of the National Kidney Foundation (NKF) and the National Institute of Diabetes and Digestive and Kidney Diseases (NIDDK)," American Journal of Kidney Diseases, vol. 42, no. 4, pp. 617-622, 2003. 


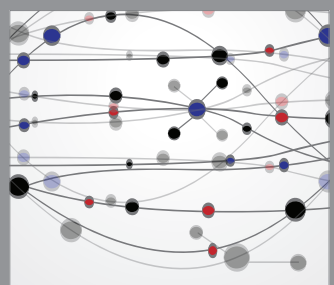

The Scientific World Journal
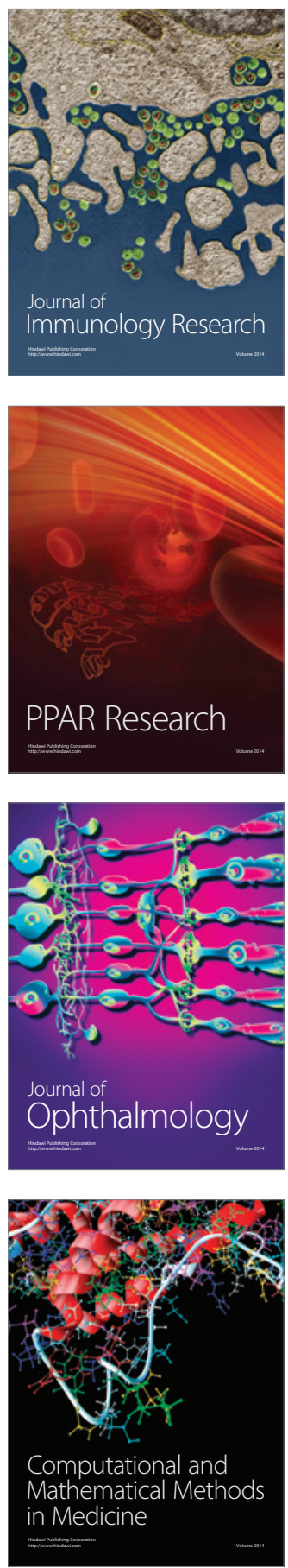

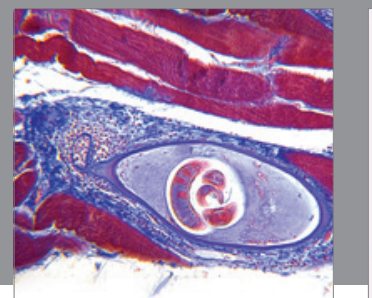

Gastroenterology

Research and Practice
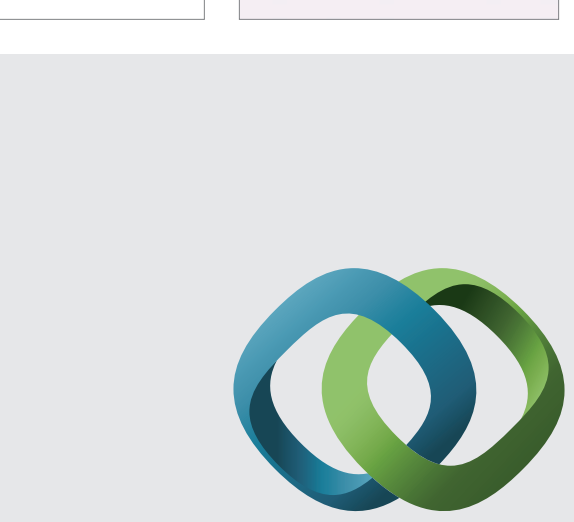

\section{Hindawi}

Submit your manuscripts at

http://www.hindawi.com
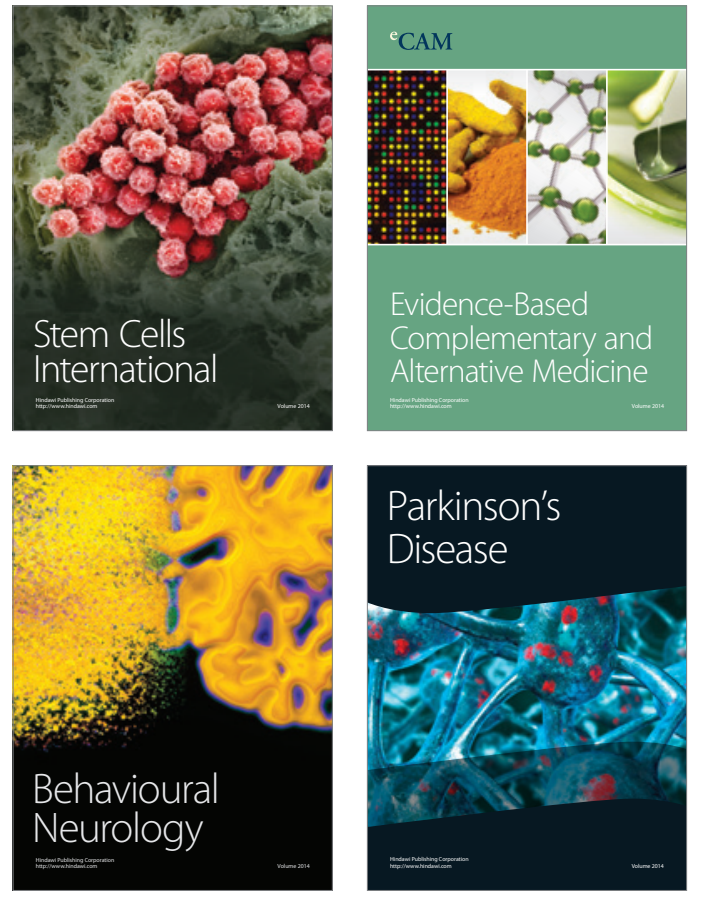
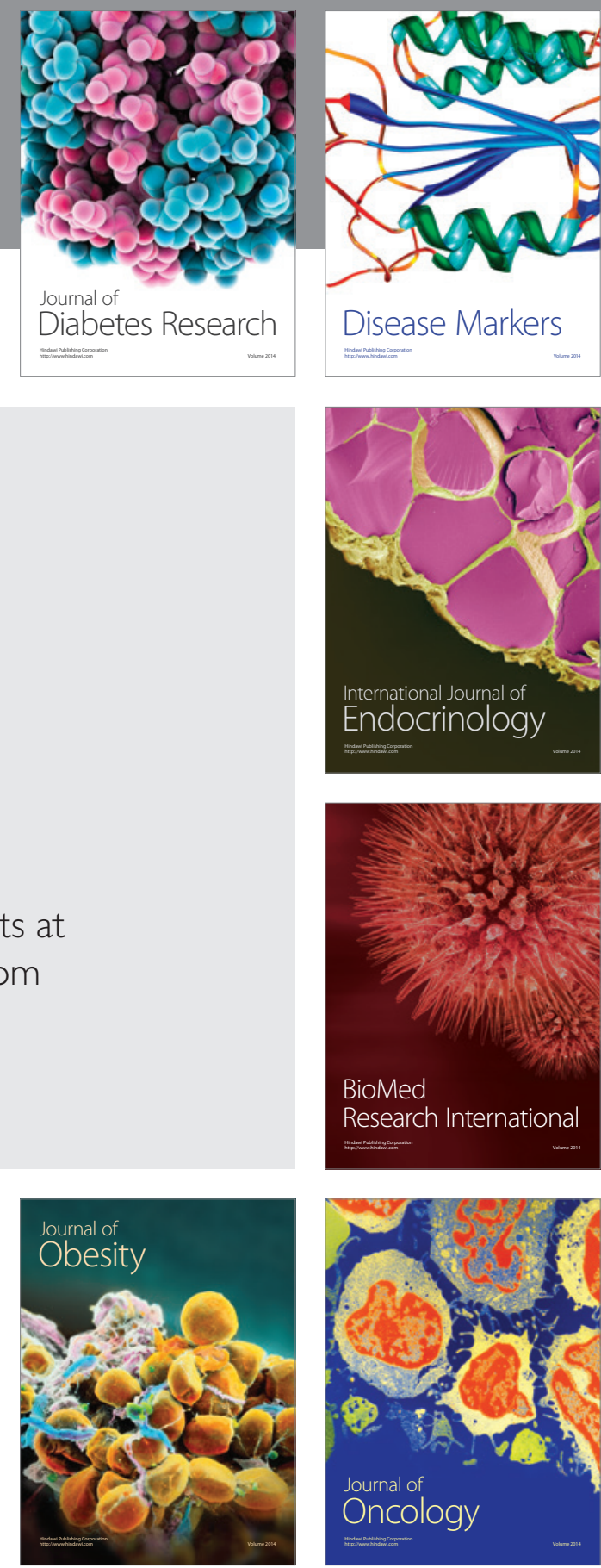

Disease Markers
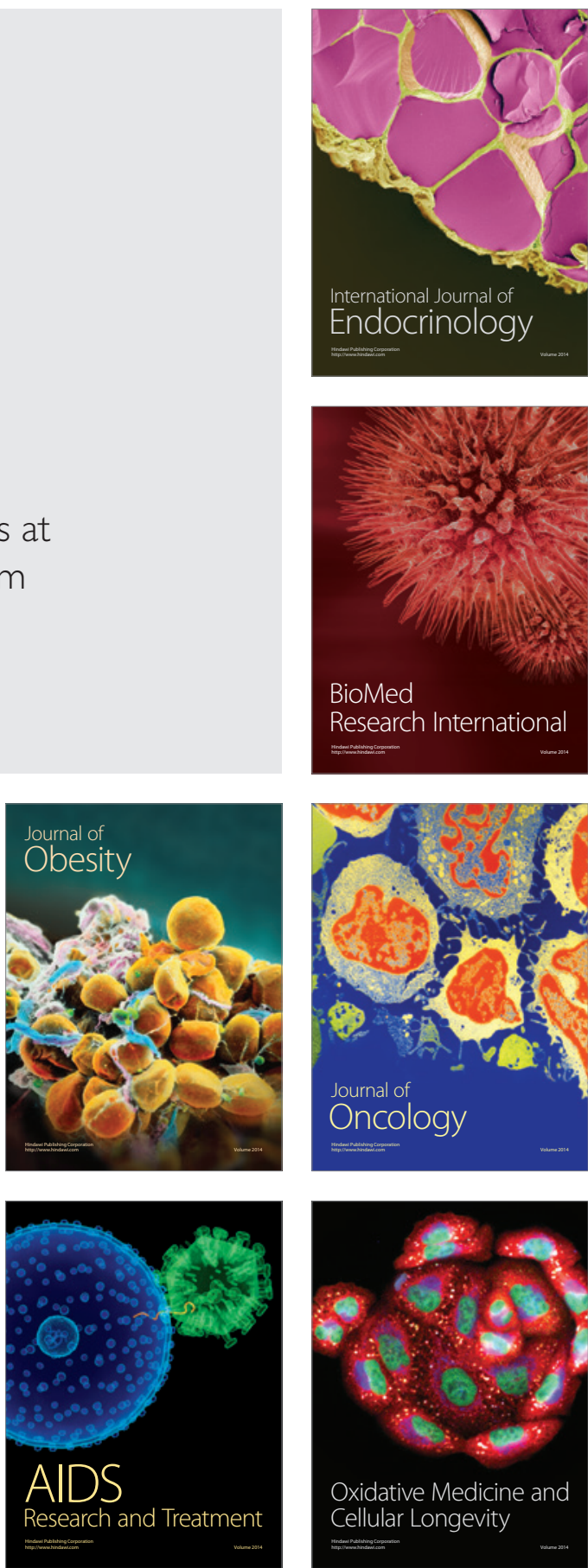